\title{
VEGFR-2 DNA Vaccine VXM01
}

National Cancer Institute

\section{Source}

National Cancer Institute. VEGFR-2 DNA Vaccine VXM01. NCI Thesaurus. Code C99378.

An orally available DNA cancer vaccine containing an attenuated strain of the bacterium Salmonella typhimurium encoding murine vascular endothelial growth factor receptor 2 (VEGFR-2) (VXM01), with potential immunomodulating, anti-angiogenic and antineoplastic activity. Upon oral administration and successful transduction, VEGFR-2 DNA vaccine VXM01 expresses VEGFR-2 in addition to inducing the expression of T-cell activation markers, such as CD25, interleukin-2, the early T-cell activation antigen CD69 and the lymphocyte function-associated antigen LFA-2. The immune response targets the fast growing VEGFR-2 expressing endothelial cells found in the tumor vasculature, thereby blocking angiogenesis which may ultimately inhibit tumor cell proliferation. VEGFR-2 is a receptor tyrosine kinase overexpressed on proliferating endothelial cells in the tumor vasculature. 